\title{
Hyperspectral Imaging as a Tool to Detect and Characterize Nanoparticles in Complex Biofluids
}

Marina Mulenos George ${ }^{1}$, Christie Sayes ${ }^{2}$ and Bernd Zechmann ${ }^{2}$

${ }^{1}$ Baylor University, Woodway, Texas, United States, ${ }^{2}$ Baylor University, Waco, Texas, United States

With recent technological advances in hyperspectral imaging, a corresponding interest in cellular uptake of nanoparticles has increased. Previously in the literature, transmission electron microscopy (TEM) was used to monitor where nanoparticles accumulate in the cell. However, this technique is labor intensive and not cost efficient for the general lab. Hyperspectral imaging is user friendly, cost effective, and the sample preparation is less labor intensive compared to TEM. Many studies in the literature show that nanoparticles of different composition, charge, or morphologies can accumulate in different cellular compartments, but these studies have been limited to engineered particles. There is an increasing need to investigate where biologically transformed nanomaterials will accumulate inside tissues and cells. Here, we studied biotransformed silver nanoparticles (AgNPs) of either positive, negative, or neutral charge in the $50 \mathrm{~nm}$ size range. Each particle system was incubated in blood serum for 24 hours at $37 \mathrm{C}$ and then characterized with transmission electron microscopy for morphological assessment and dynamic light scattering to determine particle size. Transmission electron microscopy methods included depositing a formvar coated copper grid on $10 \mu \mathrm{L}$ of suspended AgNPs for five minutes. The grid was allowed to dry and then was imaged on a JEOL JEM-1010 transmission electron microscope at 60kV. Human hepatoma cells, HepG2, were grown in a cell treated four-well chamber slide and exposed to the transformed nanoparticles for 24 hours at $37 \mathrm{C}$ with $5 \% \mathrm{CO}_{2}$.

For fluorescent imaging preparation, the cells exposed to transformed AgNPs went through fixation, permeabilization, blocking, staining with three dyes (MitoTracker Red CMXRos to stain mitochondria red, NucBlue Live ReadyProbes to stain the DNA rich nucleus blue, and ActinGreen 488 ReadyProbes to stain F-actin green), and then set with antifade mountant using the Image-iT Fixation/Permeabilization kit from ThermoFischer Scientific. (Fig. 2A). For hyperspectral imaging analyses, a CytoViva Hyperspectral Microscope was used in fluorescent mode to determine a region of interest and then in enhanced dark field mode to complete hyperspectral imaging. Hyperspectral scans had an exposure time of $0.25 \mathrm{~s}$ in full mode (600 scans per acquisition area.) Spectra were recorded of nanoparticles in the selected regions of interest and analyzed.

Our results indicate that the transformed AgNPs which released ions had an increase in cell penetration. The surface charge directly influenced the uptake mechanism in which the transformed particles entered the cell. These findings are consistent with the current literature in both in vitro and in vivo studies. (1-3) The spectral maps were significantly different from each other due to charge variation. Furthermore, the concentration of silver uptake into the cell was quantified via ICP-MS methods where the neutral particles were seen to have the largest concentration of particle or produced particle ions in the cell. The information obtained from these studies provides crucial insight into colloidal stability of nanoparticles, provides readacross comparisons between engineered and transformed metal-based particles, and aids in filling the literature gap determining how colloidal stability affects to nanoparticle biotransformation, in situ. Additionally, this technique is time and cost efficient compared to previous published methods.

The complex mixture of engineered nanomaterials in a plethora of consumer products, created by the interactions between organic matter (i.e. proteins in serum) and particle surfaces, will become more intricate as human exposure to nanomaterials increase over time. The high incidence of fatty lipids and 
carbohydrate sugars within blood circulation of aging individuals have the potential to react with metal particles to create organometallic biotransformed particles, which currently have unknown health effects. In particular, the identity, concentration, and toxicological effects of this transformed class of AgNPs should be investigated and reported. The data that presented here is of world-wide importance as environmental health scientists analyze the safety of newly created nanosilver-enabled consumer products that are on the market now and in the years to come. Consumer products must be responsibility developed and tested for safety and therefore hyperspectral imaging used as a tool to elucidate nanoparticle transformation cytotoxicity should be a priority in environmental health research.

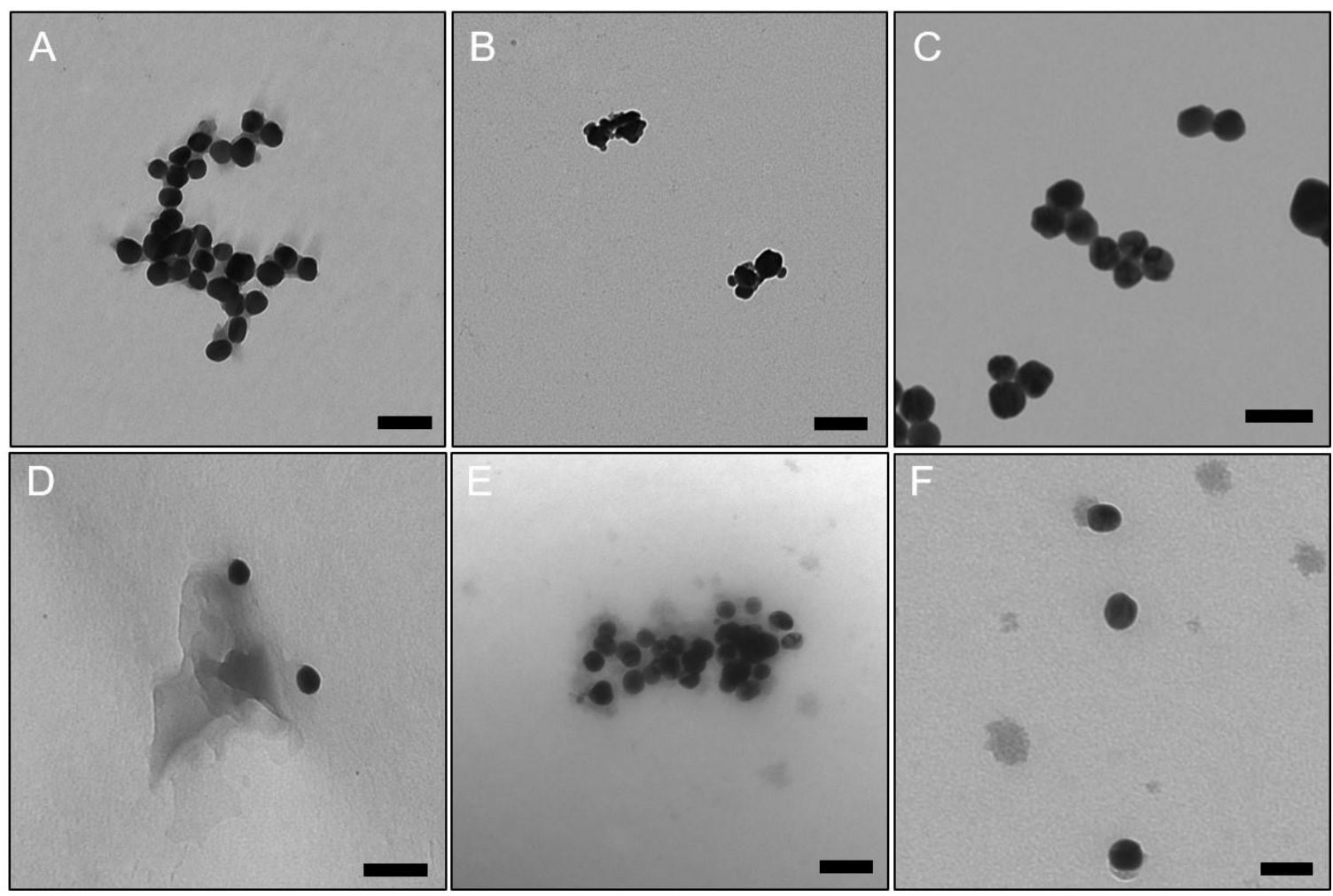

Figure 1. Transmission electron micrographs represent simulated biotransformation of silver nanoparticles. A-C are of positive, negative, and neutral surface coated particles, respectively, before biotranformation occurred. D-F represent AgNP biomolecular adsorption for positive, negative, and neutral charged particles. Scale bars represent $50 \mathrm{~nm}$.

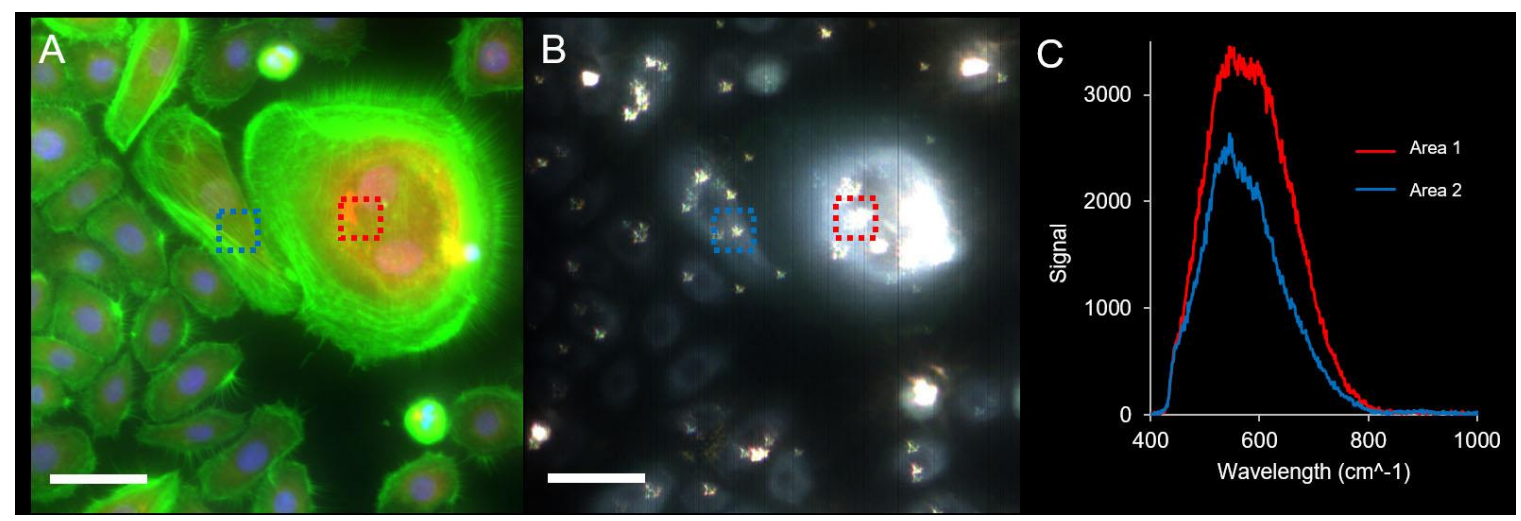

Figure 2. Hyperspectral imaging of HepG2 cells exposed to metal nanoparticles. Fluorescent imaging of the cells stained for nucleus (blue), mitochondria (red) and cytoskeleton (green) (A). This imaged was 
used to find a region of interest in the cells to scan (B) in dark field mode and collect spectra on the nanoparticles uptaken by the cell (C). Two spots were acquired to show the spectral difference between the same type of nanoparticles uptake into different cells. In spot 1 the cell shows signs of potentially increased reactive oxygen species generation (A) and in the darkfield view and spectra, the concentration of nanoparticles was seen to be larger than that of spot 2. Scale bars represent $100 \mu \mathrm{m}$.

\section{References}

1. Sun, J., Bi, C., Chan, H. M., Sun, S., Zhang, Q., \& Zheng, Y. (2013). Curcumin-loaded solid lipid nanoparticles have prolonged in vitro antitumour activity, cellular uptake and improved in vivo bioavailability. Colloids and surfaces b: biointerfaces, 111, 367-375.

2. Davda, J., \& Labhasetwar, V. (2002). Characterization of nanoparticle uptake by endothelial cells. International journal of pharmaceutics, 233(1-2), 51-59.

3. Panyam, J., Sahoo, S. K., Prabha, S., Bargar, T., \& Labhasetwar, V. (2003). Fluorescence and electron microscopy probes for cellular and tissue uptake of poly (D, L-lactide-co-glycolide) nanoparticles. International journal of pharmaceutics, 262(1-2), 1-11. 